\title{
Spanning Trees and Function Classes
}

\author{
Jeffery B. Remmel \\ Department of Mathematics \\ U.C.S.D., La Jolla, CA, 92093-0112 \\ jremmel@ucsd.edu \\ S. Gill Williamson \\ Department of Computer Science and Engineering \\ U.C.S.D., La Jolla, CA, 92093-0114 \\ gwilliamson@ucsd.edu
}

Submitted September 17, 2001; Accepted August 12, 2002

MR Subject Classifications: 05A15, 05C05, 05C20, 05C30

\begin{abstract}
If $G=K_{n}$ is the complete graph, the classical Prüffer correspondence gives a natural bijection between all spanning trees of $G$ (i.e., all Cayley trees) and all functions from a set of $n-2$ elements to a set of $n$ elements. If $G$ is a complete multipartite graph, then such bijections have been studied by Ĕgecioğlu and Remmel. In this paper, we define a class of directed graphs, called filtered digraphs, and describe a natural class of bijections between oriented spanning forests of these digraphs and associated classes of functions. We derive multivariate generating functions for the oriented spanning forests which arise in this context, and we link basic properties of these spanning forests to properties of the functions to which they correspond. This approach yields a number of new results for directed graphs. Moreover, in the undirected case, various specializations of our multivariate generating function not only include various known results but also give a number of new results.
\end{abstract}

\section{Introduction}

This paper is motivated by the work of Ĕgecioğlu and Remmel [4] who gave a bijective proof of the formula $n^{n-2}$ for the number of Cayley trees on $n$ vertices, i.e. the number of spanning trees of the complete graph $K_{n}$. In particular, they showed that there is a natural bijection between the set of $C_{n, 1}$ of all Cayley trees on $n$ vertices where all edges are directed toward the root 1 and the class of functions $\mathcal{F}=\{f:\{2, \ldots, n-1\} \rightarrow\{1, \ldots, n\}\}$. Later in [5], Eğecioğlu and Remmel extended this idea to give a bijective proof for the number of spanning trees of the complete $k$-partite graph, $K_{n_{1}, \ldots, n_{k}}$. Again in [5], Eŭgecioğlu 
and Remmel showed that there was a natural bijection between a certain class of functions $f:\{2, \ldots, n-1\} \rightarrow\{1, \ldots, n\}$ and the set of spanning trees of $K_{n_{1}, \ldots, n_{k}}$ rooted at vertex 1 .

It is well known that the formulas for the number of spanning trees of $K_{n}$ and $K_{n_{1}, \ldots, n_{k}}$ follow from the matrix tree theorem [1]. One advantage of [4, 5] over the matrix tree theorem approach is that the resulting bijections give rise to natural multivariate generating functions which keep track of the descent and rise edges for the set of root-directed spanning trees, i.e., the spanning trees where all edges are directed toward the root. A second advantage of the bijective approach of $[4,5]$ is that there are well known techniques $[10,11,12]$ for ranking and unranking function classes and hence the bijections provide ways to rank and unrank spanning trees of $K_{n}$ and $K_{n_{1}, \ldots, n_{k}}$.

In this paper, we define a class of directed graphs, called filtered digraphs, and describe a natural class of bijections between oriented spanning forests of these digraphs and associated classes of functions. We derive multivariate generating functions for the oriented spanning forests which arise in this context and we link basic properties of these spanning forests to properties of the functions to which they correspond. We should note that the class of filtered digraphs contains not only both $K_{n}$ and $K_{n_{1}, \ldots, n_{k}}$ but also many directed graphs to which the matrix tree theorem does not apply. Thus we extend the methods of Eğecioğlu and Remmel to a much larger class of graphs. In addition, we extend the results of Ĕgecioğlu and Remmel in two other ways. First, our methods apply to spanning forests rather than just to spanning trees. Second, our multivariate generating functions are finer than those considered by Ĕgecioğlu and Remmel and hence have a greater variety of specializations.

This paper is organized as follows. In section two, we define the class of filter digraphs and their corresponding function classes. We then define the bijection between the function class of a filtered digraph and the set of root-directed spanning trees of the filtered digraph. Our main result is Theorem 2.4 where we prove the validity of this bijection and show how the bijection allows us to derive a multivariate generating function for the set of root-directed spanning forests of a filtered digraph. In section three, we give three examples.

Example 3.1. We consider the case when $G=K_{n}$. Our method gives a new multivariate generating function for the set of rooted spanning forests of $K_{n}$. In addition, we show that certain specializations of our multivariate generating function allow us to derive new formulas for the number of spanning forests with specified sets of ascent and descent edges.

Example 3.2. We show that similar results hold for the root-directed spanning forests of $K_{n_{1}, \ldots, n_{k}}$.

Example 3.3. We show how similar formulas can be derived for a basic class of multipartite cyclic digraphs. These results are new and are not covered by the classical matrix tree theorem. 
We end section three with a brief discussion of some additional classes of filtered digraphs $G$ for which one can derive closed expressions for the generating functions for the set of root-directed spanning forests of $G$.

\section{General results for directed graphs}

In this section, we shall introduce the definition of a filtered digraph and prove our main result.

Let $[n]=\{1,2, \ldots, n\}$. Let $G=([n], E)$ be a digraph with vertex set $[n]$ and edge set $E$. Let $\mathbf{F}=\left(c_{1}, c_{2}, \ldots, c_{k}\right)$ be a composition of $n$. That is, assume $c_{i}$ is a positive integer for each $i$ and $\sum_{i=1}^{k} c_{i}=n$. Let $N_{0}=0$ and let $N_{t}=c_{1}+\cdots+c_{t}$ for $t=1, \ldots, k$. We let $\mathcal{C}_{t}=\left\{1+N_{t-1}, \ldots, N_{t}\right\}$ for $t=1, \ldots, n$. Note that each $\mathcal{C}_{t}$ is an interval and the collection of nonempty sets $\left\{\mathcal{C}_{i} \mid i=1, \ldots, k\right\}$ forms a set partition of $[n]$. We call this set partition the filtration associated with the composition $\mathbf{F}$.

Definition 2.1 Given a composition $\mathbf{F}=\left(c_{1}, \ldots, c_{k}\right)$ of $n$, we define a partial order relation $\leq_{\mathbf{F}}$ on $[n]$ by $x \leq_{\mathbf{F}} y$ if $x=y$ or if $x \in \mathcal{C}_{i}$ and $y \in \mathcal{C}_{j}$ where $1 \leq i<j \leq k$. We call $\leq_{\mathbf{F}}$ the filtration order on $[n]$.

We write $x<_{F} y$ if $x \leq_{F} y$ but $x \neq y$. If $x<_{F} y$, then our definitions ensure that $x<y$ as integers. Note that $1 \in C_{1}, n \in C_{k}$, and each of the sets $\mathcal{C}_{i}$ is a set of incomparable elements (i.e., an antichain) with respect to $\leq_{F}$. In the standard terminology for posets, $\leq_{\mathbf{F}}$ is the ordinal sum of the antichains $\mathcal{C}_{i}, 1 \leq i \leq k$.

Definition 2.2 Let $\left\{\mathcal{C}_{i}: i=1, \ldots, k\right\}$ be the filtration associated with the composition $\mathbf{F}=\left(c_{1}, \ldots, c_{k}\right)$ of $n$. Let $I_{B}$ and $I_{S}$ be subsets of $[k]$ and let $\mathcal{B}=\left\{C_{i}: i \in I_{B}\right\}$ and $\mathcal{S}=\left\{C_{i}: i \in I_{S}\right\}$. We refer to the sets $\mathcal{B}$ and $\mathcal{S}$ as the bases and summits of $G$ respectively. $A$ set $\mathcal{C}_{i} \in \mathcal{B}$ is called a base of $G$ and its elements are called base vertices of $G$. A set $\mathcal{C}_{i} \in \mathcal{S}$ is called a summit of $G$ and its elements are called summit vertices of $G$. We say that a digraph $G=([n], E)$ is a filtered digraph with respect to $\mathbf{F}, I_{B}$, and $I_{S}$, if the following conditions hold for all $x, y \in[n]$.

1. $1 \in I_{B}, 1 \notin I_{S}, k \notin I_{B}$, and $k \in I_{S}$.

2. If $x, y \in \mathcal{C}_{i}$ for some $1 \leq i \leq k$, then $(x, y) \notin E$.

3. If $y<_{F} x$, then $(x, y) \in E$ if and only if there exist $p<q$ such that $x \in C_{q}, q \in I_{S}$, $y \in C_{p}$ and $p \in I_{B}$.

4. If $x \in \mathcal{C}_{i}, 1 \leq i<k$, and $\mathcal{C}_{i}$ is not a summit, then there is some $y$ such that $(x, y) \in E$ and $x<_{\mathbf{F}} y$.

It is perhaps helpful to paraphrase conditions (1)-(4). Condition (1) states that $\mathcal{C}_{1}$ is a base but not a summit and $\mathcal{C}_{k}$ is a summit but not a base. Otherwise the bases and summits are arbitrary. A set $\mathcal{C}_{i}$ with $i \notin\{1, k\}$ may be both a base and a summit. 
Condition (2) states that the restrictions of $G$ to the sets $\mathcal{C}_{i}$ are empty digraphs (no edges). Condition (3) states that all directed edges between summit vertices and "lower" base vertices are present and these are the only "downward" edges in $E$. That is, these are the only edges $(x, y) \in E$ with $y<_{\mathbf{F}} x$. Finally, condition (4) states that for any vertex $x$ that does not belong to a summit, there is at least one upward edge out of $x$. That is, there is at least one edge $(x, y) \in E$ such that $x<_{\mathbf{F}} y$.

Given any digraph $G^{\prime}=\left([n], E^{\prime}\right)$, we can define the set of "root-directed" spanning forests of $G^{\prime}$ with roots $r_{1}, \ldots, r_{q} \in[n]$ as follows. First we regard the digraph $G^{\prime}$ as an undirected graph in the obvious manner. Next we consider the set of all spanning forests $T^{\prime}=\left(T_{1}^{\prime}, \ldots, T_{q}^{\prime}\right)$ of this undirected version of $G^{\prime}$ with subtrees $T_{i}^{\prime}, i=1, \ldots, q$, such that $r_{i} \in T_{i}^{\prime}$ for $i=1, \ldots, q$. We can then think of each $T_{i}^{\prime}$ as a directed graph by considering $r_{i}$ as the root of $T_{i}^{\prime}$ and directing all edges back toward the root. That is, we direct all edges in $T_{i}^{\prime}$ so that there is a directed path from each vertex $v$ in $T_{i}^{\prime}$ to $r_{i}$. If for each $i$, all these directed edges are in fact in $E$, then we say that $T^{\prime}$ is a root-directed spanning forest of $G^{\prime}$ with roots $r_{1}, \ldots, r_{q}$. Alternatively, such spanning forests are called "oriented," but we shall stick to the former terminology.

We denote the set of all root-directed spanning forests of $G^{\prime}$ with roots $r_{1}, \ldots, r_{q}$ by $\mathcal{T}_{\left\{r_{1}, \ldots, r_{q}\right\}}^{G^{\prime}}$. If $n \notin\left\{r_{1}, \ldots, r_{q}\right\}$, then we use the notation $\mathcal{T}_{\left\{r_{1}, \ldots, r_{q}\right\} ; r_{j}}^{G^{\prime}}$ to designate all root-directed spanning forests of $G^{\prime}$ with $n$ in the component tree rooted at $r_{j}$.

Returning to the case $G=([n], E)$, suppose we are given a directed edge $(i, j)$ where $1 \leq i, j \leq n$. Following a suggestion of Peter Doyle [3], we define the weight of $(i, j)$, $W((i, j))$, by

$$
W((i, j))=\left\{\begin{array}{l}
p_{i} s_{j} \text { if } i<j, \\
q_{i} t_{j} \text { if } i \geq j
\end{array}\right.
$$

where $p_{i}, q_{i}, s_{i}, t_{i}$ are variables for $i=1, \ldots, n$. We shall call a directed edge $(i, j)$ a descent edge if $i \geq j$ and an ascent edge if $i<j$. We then define the weight of any digraph $G=([n], E)$ by

$$
W(G)=\prod_{(i, j) \in E} W((i, j))
$$

Definition 2.3 Let $G=([n], E)$ be a filtered digraph with respect to the composition $\mathbf{F}=\left(c_{1}, \ldots, c_{k}\right)$, the set of bases indexed by $I_{B}$ and the set of summits indexed by $I_{S}$. Let $m$ be such that $1 \leq m<n-1$ and assume that $1, \ldots, m$ are base vertices of $G$. Suppose that $m \in \mathcal{C}_{t}$. Let $\mathcal{F}_{n}(G, \mathbf{F}, m)$ be the set of all functions $f:\{m+1, \ldots, n-1\} \rightarrow[n]$ that satisfy the following conditions.

1. If $f(i) \neq i$ then $(i, f(i)) \in E$.

2. If $f(i)=i$ and $i \in \mathcal{C}_{p}$, then $p \in I_{S} \cap I_{B}$ and $p \geq t$.

3. If $p \in I_{S} \cap I_{B}$ and $p \geq t$, then there is at most one $i \in \mathcal{C}_{p}$ such that $f(i)=i$.

We call $\mathcal{F}_{n}(G, \mathbf{F}, m)$ the $m$-canonical function class for the filtered digraph $G$ with respect to $\mathcal{F}, I_{B}$ and $I_{S}$. 
We note that our conditions ensure that $\mathcal{F}_{n}(G, \mathbf{F}, m)$ is not empty. That is, suppose that $v \in[n]-\{n, 1, \ldots m\}$ is in $\mathcal{C}_{i}$. Now if $\mathcal{C}_{i}$ is a summit, then we know that $i>1$. Moreover, we know that $1 \in \mathcal{C}_{1}$ and that $\mathcal{C}_{1}$ is a base so that $(v, 1)$ in $E$. Thus there is at least one choice for $f(v)$. Similarly, if $\mathcal{C}_{i}$ is not a summit, then $i<k$ and we know that there is at least one upward edge out of $v$ in $G$. Thus again, there is at least one choice for $f(v)$.

We can think of each $f \in \mathcal{F}_{n}(G, \mathbf{F}, m)$ as a directed graph on the vertex set $\{1, \ldots, n\}$. That is, if $f(i)=j$, then there is a directed edge from $i$ to $j$. A moment's thought will convince one that, in general, the digraph corresponding to a function $f \in \mathcal{F}_{n}(G, \mathbf{F}, m)$ will consists of $m+1$ root-directed trees rooted at vertices $1, \ldots, m$ and $n$ respectively, with all edges directed toward their roots, plus a number of directed cycles of length $\geq 1$. For each vertex $v$ on a given cycle, there is possibly a root-directed tree attached to $v$ with $v$ as the root and all edges directed toward $v$. Note the fact that there are trees rooted at vertices $n, 1, \ldots, m$ is due to the fact that these elements are not in the domain of $f$. Thus there can be no directed edges out of any of these vertices. We let the weight of $f, W(f)$, be the weight of the digraph associated with $f$.

Suppose that we are given a filtered digraph $G=([n], E)$ with respect to the composition $\mathbf{F}=\left(c_{1}, \ldots, c_{k}\right)$. Suppose that the summits are indexed by $I_{S}$ and the bases are indexed by $I_{B}$. Suppose also that $1, \ldots, m$ are fixed base elements of $G$. Let $\left\{\mathcal{C}_{i} \mid i=1, \ldots, k\right\}$ be the filtration partition for $\mathbf{F}$ and suppose that $m \in \mathcal{C}_{t}$. Thus $N_{t-1}<m \leq N_{t}$. Let $\mathcal{T}_{[m] ; j}^{G}$ denote all root-directed spanning forests of $G$ with roots in $[m]=\{1, \ldots, m\}$ and for which $n$ is a vertex of the tree (component) rooted at $j$. We shall show that in this situation, if the root $j \notin \mathcal{C}_{t}$, then there is a natural bijection $\Theta_{j}$ between the $m$-canonical function class $\mathcal{F}_{n}(G, \mathbf{F}, m)$ for $G$ and the set $\mathcal{T}_{[m] ; j}^{G}$. If $j \in \mathcal{C}_{t}$, then there is a corresponding bijection $\Theta_{j}^{*}$ from the subset $\mathcal{F}_{n}^{*}(G, \mathbf{F}, m)$ of $\mathcal{F}_{n}(G, \mathbf{F}, m)$ to $\mathcal{T}_{[m] ; j}^{G}$ where $\mathcal{F}_{n}^{*}(G, \mathbf{F}, m)$ consists of all $f \in \mathcal{F}_{n}(G, \mathbf{F}, m)$ such that $f(i) \neq i$ for all $i \in \mathcal{C}_{t}$ such that $i>m$. The fact that $\Theta_{j}^{*}$, which is simply the restriction of $\Theta_{j}$ to $\mathcal{F}_{n}^{*}(G, \mathbf{F}, m)$, is a bijection will easily follow from our proof that $\Theta_{j}$ is a bijection.

Theorem 2.4 Let $G=([n], E)$ be a filtered digraph with respect to the composition $\mathbf{F}=$ $\left(c_{1}, \ldots, c_{k}\right)$, the set of summits indexed by $I_{S}$, and the set of bases indexed by $I_{B}$. Assume that $1, \ldots, m$ are base elements of $G$, that $m \in \mathcal{C}_{t}$, and that $N_{t-1}=c_{1}+\cdots+c_{t-1}$. Then, for each $1 \leq j \leq N_{t-1}$, there is a bijection $\Theta_{j}: \mathcal{F}_{n}(G, \mathbf{F}, m) \rightarrow \mathcal{T}_{[m] ; j}^{G}$ and, for each $N_{t-1}+1 \leq j \leq m$, there is a bijection $\Theta_{j}^{*}: \mathcal{F}_{n}^{*}(G, \mathbf{F}, m) \rightarrow \mathcal{T}_{[m] ; j}^{G}$ such that

$$
\begin{aligned}
& q_{n} t_{j} W(f)=W\left(\Theta_{j}(f)\right), 1 \leq j \leq N_{t-1} \text { and } \\
& q_{n} t_{j} W(f)=W\left(\Theta_{j}^{*}(f)\right), N_{t-1}+1 \leq j \leq m .
\end{aligned}
$$

Hence

$$
q_{n}\left(t_{1}+\cdots+t_{N_{t-1}}\right) \sum_{f \in \mathcal{F}_{n}} W(f)+q_{n}\left(t_{N_{t-1}+1}+\cdots+t_{m}\right) \sum_{f \in \mathcal{F}_{n}^{*}} W(f)=\sum_{T \in \mathcal{T}_{[m]}^{G}} W(T) .
$$

Here if $t=1$, then $N_{t-1}=N_{0}=0$ and we take $t_{1}+\cdots+t_{N_{t-1}}=0$. 
Proof: To define the bijection $\Theta_{j}, 1 \leq j \leq N_{t-1}$, we first imagine that the directed graph corresponding to $f \in \mathcal{F}_{n}(G, \mathbf{F}, m)$ is drawn in two parts (see Figure 1). The first part of the graph consists of the rooted-trees at roots $1, \ldots, j-1, j+1, \ldots m$. The second part of the graph is drawn so that

(a) the trees rooted at $n$ and $j$ are drawn on the extreme left and the extreme right respectively with their edges directed upwards,

(b) the cycles are drawn so that their vertices form a directed path on the line between $n$ and $j$, with one back edge above the line, and the root-directed tree attached to any vertex on a cycle is drawn below the line between $n$ and 1 with its edges directed upwards,

(c) each cycle is arranged so that its maximum element is on the right, and

(d) the cycles are arranged so that if the maximum element $m_{c}$ of a cycle $c$ is in $\mathcal{C}_{i}$ and the maximum element $m_{c^{\prime}}$ of a cycle $c^{\prime}$ in $\mathcal{C}_{j}$, then $c$ is to the left of $c^{\prime}$ if either (i) $i>j$, (ii) $i=j$ and $c$ is a one cycle or (iii) $i=j$, neither $c$ nor $c^{\prime}$ are one cycles and $m_{c}>m_{c^{\prime}}$.

Figure 1 pictures a function $f$ drawn according to the rules (a)-(d) where $n=27$, $\mathbf{F}=(5,5,3,6,8)$, and $G=([n], E)$ is the filtered digraph defined as follows. Since $\mathbf{F}=(5,5,3,6,8), \mathcal{C}_{1}=\{1, \ldots, 5\}, \mathcal{C}_{2}=\{6, \ldots, 10\}, \mathcal{C}_{3}=\{11, \ldots, 13\}, \mathcal{C}_{4}=\{14, \ldots, 19\}$ and $\mathcal{C}_{5}=\{20, \ldots, 27\}$. We let $I_{B}=\{1,2,4\}$ and $I_{S}=\{2,4,5\}$ so that the sets $\mathcal{C}_{1}, \mathcal{C}_{2}$ and $\mathcal{C}_{4}$ are bases and the sets $\mathcal{C}_{2}, \mathcal{C}_{4}$ and $\mathcal{C}_{5}$ are summits. Finally, we specify the edges of $G$ as follows.

$\mathcal{C}_{1}: \mathcal{C}_{2}, \mathcal{C}_{3}, \mathcal{C}_{4}$

$\mathcal{C}_{2}: \mathcal{C}_{1}, \mathcal{C}_{3}, \mathcal{C}_{4}, \mathcal{C}_{5}$

$\mathcal{C}_{3}: \mathcal{C}_{4}, \mathcal{C}_{5}$

$\mathcal{C}_{4}: \mathcal{C}_{1}, \mathcal{C}_{2}, \mathcal{C}_{5}$

$\mathcal{C}_{5}: \mathcal{C}_{1}, \mathcal{C}_{2}, \mathcal{C}_{4}$

In the above specification of the edges of $G$, we interpret $\mathcal{C}_{i}: \mathcal{C}_{j_{1}}, \ldots \mathcal{C}_{j_{s}}$ to mean that there is a directed edge from every vertex $v \in \mathcal{C}_{i}$ to every vertex $w$ in $\mathcal{C}_{j_{k}}$ for $k=1, \ldots, s$.

This given, suppose that the digraph of $f$ is drawn as described above and the cycles of fare $c_{1}(f), \ldots, c_{a}(f)$ reading from left to right. We let $r_{c_{i}(f)}$ and $l_{c_{i}(f)}$ denote the right and left endpoints of the cycle $c_{i}(f)$ for $i=1, \ldots, a$. Note that if $c_{i}(f)$ is a 1-cycle, then we let $r_{c_{i}(f)}=l_{c_{i}(f)}$ be the element in the 1-cycle. $\Theta_{j}(f)$ is obtained from $f$ by simply deleting the back edges $\left(r_{c_{i}(f)}, l_{c_{i}(f)}\right)$ for $i=1, \ldots, a$ and adding the directed edges $\left(r_{c_{i}(f)}, l_{c_{i+1}(f)}\right)$ for $i=1, \ldots, a-1$ plus the directed edges $\left(n, l_{c_{1}(f)}\right)$ and $\left(r_{c_{a}(f)}, j\right)$. That is, we remove the all the back edges that are above the line, and then we connect $n$ to the lefthand endpoint of the first cycle, the righthand endpoint of each cycle to the lefthand endpoint of the cycle following it, and we connect the righthand endpoint of the last cycle to $j$. For example, $\Theta_{2}(f)$ is pictured in Figure 2 for the $f$ given in Figure 1. If there are no cycles 


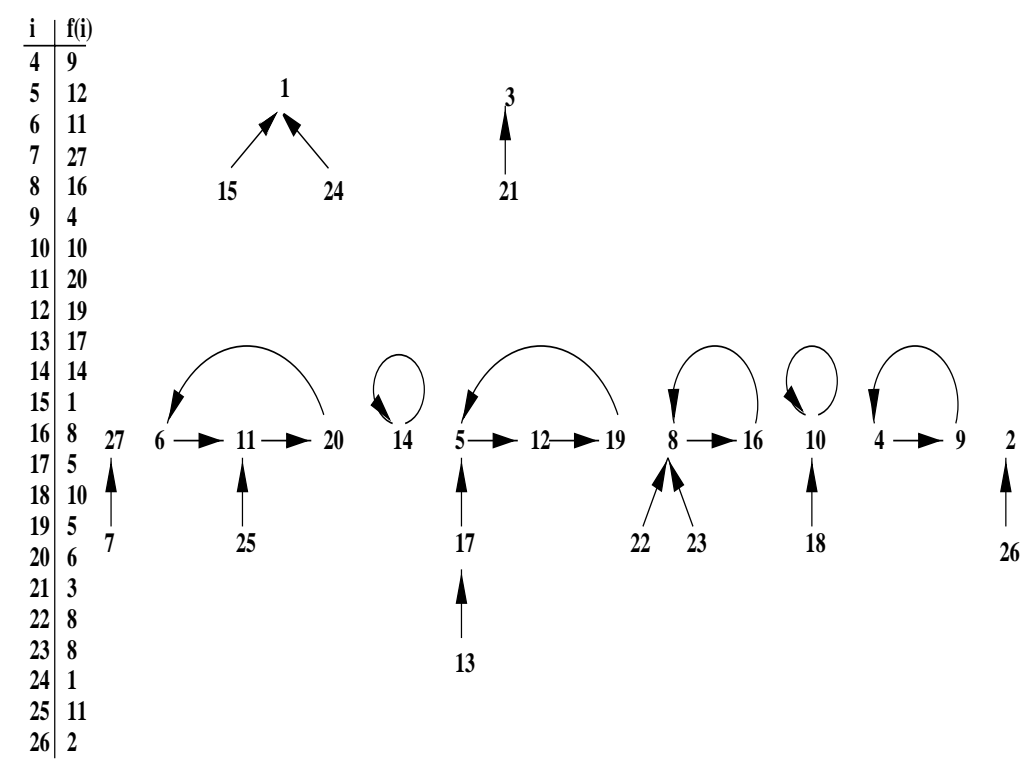

Figure 1: The digraph of a function

in $f$, then $\Theta_{j}(f)$ is simply the result of adding the directed edge $(n, j)$ to the digraph of $f$.

To see that $\Theta_{j}(f) \in \mathcal{T}_{[m] ; j}^{G}$ for all $f \in \mathcal{F}_{n}(G, \mathbf{F}, m)$, first observe that for all $i=1, \ldots, a$, $l_{c_{i}(f)}$ must be a base element and $r_{c_{i}(f)}$ must be a summit element. That is, if $c_{i}$ is a cycle of length one, then $f\left(r_{c_{i}(f)}\right)=l_{c_{i}(f)}=r_{c_{i}(f)}$ so that $l_{c_{i}(f)}$ must be both a summit and base element, since by our definition of $\mathcal{F}_{n}(G, \mathbf{F}, m)$, the only fixed points of $f$ are elements of $\mathcal{C}_{p}$ for some $p \in I_{B} \cap I_{S}$. If $c_{i}$ is not a one cycle, then $f\left(r_{c_{i}(f)}\right)=l_{c_{i}(f)}<r_{c_{i}(f)}$ since $r_{c_{i}(f)}$ is the largest element of $c_{i}$. By our definition of $\mathcal{F}_{n}(G, \mathbf{F}, m),\left(r_{c_{i}(f)}, l_{c_{i}(f)}\right)$ must be a downward edge of $G$ so that $r_{c_{i}(f)}$ must be a summit element and $l_{c_{i}(f)}$ must be a base element. In particular, this means that the edges $\left(n, l_{c_{1}(f)}\right)$ and $\left(r_{c_{a}(f)}, j\right)$ are elements of $G$. Recall here that $n \in \mathcal{C}_{k}$, which is the highest summit, and hence $n$ is connected to all base elements in $G$. On the other hand, by assumption, $j \in\left[N_{t-1}\right] \subseteq[m]$. By definition, $1, \ldots, m$ are base elements and $m \in \mathcal{C}_{t}$. Thus $\{1, \ldots, m\} \subseteq \cup_{b=1}^{t} \mathcal{C}_{b}$ and $\mathcal{C}_{1}, \ldots, \mathcal{C}_{t}$ are all bases. Now suppose that $r_{c_{a}(f)} \in \mathcal{C}_{s}$. There are two possibilities. First it could be that $c_{a}$ is a one cycle. Then in this case, our definition of $\mathcal{F}_{n}(G, \mathbf{F}, m)$ ensures that $s \in I_{B} \cap I_{S}$ and $s \geq t$. Thus the edge $\left(r_{c_{a}(f)}, j\right)$ connects a summit vertex to an element in a lower base and hence is in $G$. If $c_{a}$ is not a one cycle, $l_{c_{a}(f)} \in \mathcal{C}_{u}$ where $u \in I_{B}$ and $u<s$. However, since $l_{c_{a}(f)}$ is part of a cycle of $f$ (hence in the domain of $f$ ), we know that $l_{c_{a}(f)}>m$. Thus, by our definition of a filtration, we know that $u \geq t$ since $m \in \mathcal{C}_{t}$. Since $j \in \mathcal{C}_{r}$ for some $r<t$ and $j$ is a base element, $\mathcal{C}_{r}$ is a base below the summit $\mathcal{C}_{s}$ so that again we can conclude that $\left(r_{c_{a}(f)}, j\right)$ is in $G$.

Now consider the other edges $\left(r_{c_{i}(f)}, l_{c_{i+1}(f)}\right)$ that we added to the digraph of $f$. There are two cases to consider.

Case $1 c_{i+1}$ is a one cycle. 

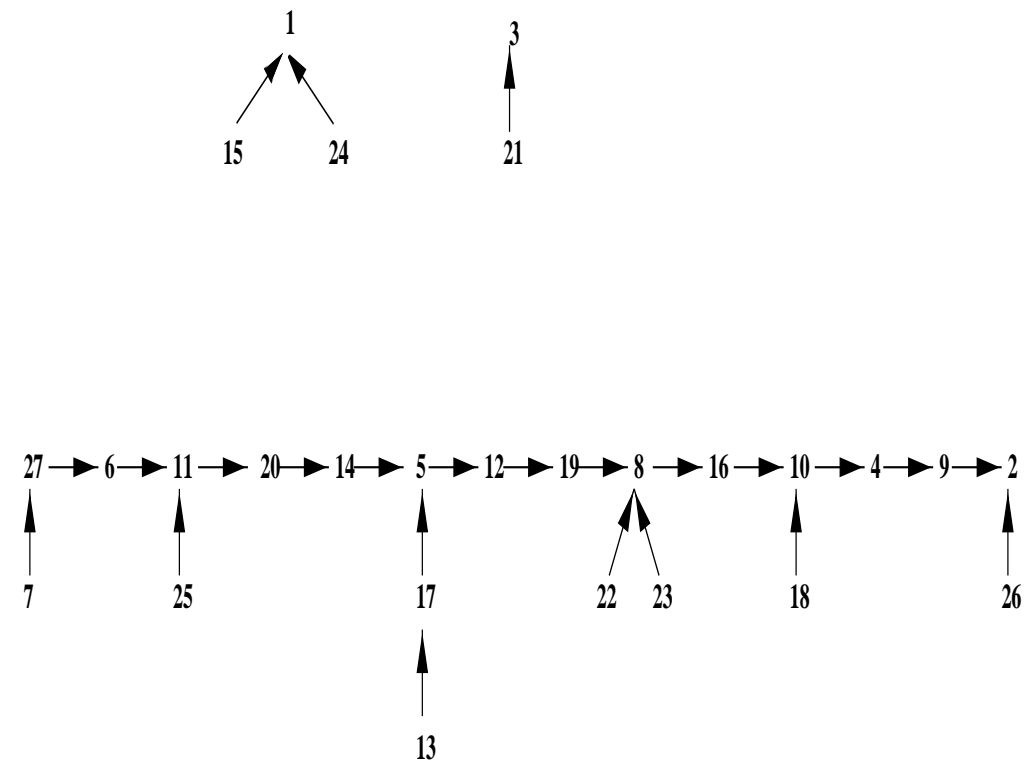

Figure 2: $\Theta_{2}(f)$

Then $l_{c_{i+1}(f)}=r_{c_{i+1}(f)} \in \mathcal{C}_{p}$ for some $p \in I_{B} \cap I_{S}$. However, by our convention for drawing the digraph of $f, c_{i+1}$ must be the leftmost cycle of the form $c_{k}$ such that $r_{c_{k}(f)} \in \mathcal{C}_{p}$. Hence $r_{c_{i}(f)}$ must be in some $\mathcal{C}_{w}$ where $w>p$. Since $r_{c_{i}(f)}$ is a summit element, the edge $\left(r_{c_{i}(f)}, l_{c_{i+1}(f)}\right)$ goes from a summit vertex to a vertex which is in a lower base and hence is in $G$.

Case $2 c_{i+1}$ is not a one cycle.

Then $l_{c_{i+1}(f)}<r_{c_{i+1}(f)}$. Thus $r_{c_{i+1}(f)} \in \mathcal{C}_{p}$ for some $p \in I_{S}$ and $l_{c_{i+1}(f)} \in \mathcal{C}_{s}$ for some $s \in I_{B}$ where $s<p$ since $\left(r_{c_{i+1}(f)}, l_{c_{i+1}(f)}\right)$ is a downward edge in $G$. By our convention for ordering the cycles, we know that $r_{c_{i}(f)} \in \mathcal{C}_{u}$ where $u \geq p$. Thus $\mathcal{C}_{u}$ must be a summit which lies above the base $\mathcal{C}_{s}$ so again the edge $\left(r_{c_{i}(f)}, l_{c_{i+1}(f)}\right)$ must be in $G$.

In the special case where $f$ has no cycles, we add only the edge $(n, j)$ which must be in $G$ since $j$ is in a base which lies below the top summit $\mathcal{C}_{k}$. It follows that all the new edges that we add to the digraph of $f$ are in $G$. Note that since we remove all the backedges and these are the only possible loops in the digraph of $f$, all the remaining edges are of the form $(i, j)$ where $f(i)=j$ and $i \neq j$ and hence are in $G$ by our definition of $\mathcal{F}_{n}(G, \mathbf{F}, m)$. Thus $\Theta_{j}(f) \in \mathcal{T}_{[m] ; j}^{G}$ for all $f \in \mathcal{F}_{n}(G, \mathbf{F}, m)$.

The weight preserving property of $\Theta_{j}$ is also easy to verify. That is, by our conventions, any backedges $\left(r_{c_{i}(f)}, l_{c_{i}(f)}\right)$ are descent edges so that its weight is $q_{r_{c_{i}(f)}} t_{l_{c_{i}(f)}}$. Thus the total weight of the backedges is

$$
\prod_{i=1}^{a} q_{r_{c_{i}(f)}} t_{l_{c_{i}(f)}} .
$$

Our argument above shows that all the new edges that we add are also descent edges so 
that the weight of the new edges is

$$
q_{n} t_{l_{c_{1}(f)}}\left(\prod_{i=1}^{a-1} q_{r_{c_{i}(f)}} t_{l_{c_{i+1}(f)}}\right) q_{r_{c_{a}(f)}} t_{j}=q_{n} t_{j} \prod_{i=1}^{a} q_{r_{c_{i}(f)}} t_{l_{c_{i}(f)}} .
$$

Since all the remaining edges have the same weight in both the digraph of $f$ and in the digraph $\Theta_{j}(f)$, it follows that $q_{n} t_{j} W(f)=W\left(\Theta_{j}(f)\right)$ as claimed.

To see that $\Theta_{j}$ is a bijection, we shall describe how to define $\Theta_{j}^{-1}$. Given a forest $T \in \mathcal{T}_{[m]: j}^{G}$, consider the path

$$
m_{0}=n, x_{1}, \ldots, m_{1}, x_{2}, \ldots m_{2}, \ldots, x_{t}, \ldots, m_{t}, j
$$

where $m_{i}$ is the maximum interior vertex on the path from $m_{i-1}$ to $j, 1 \leq i \leq t$. If $\left(m_{i-1}, m_{i}\right)$ is an edge on this path, then it is understood that $x_{i}, \ldots, m_{i}=m_{i}$ consists of just one vertex and we define $x_{i}=m_{i}$. Note that by definition $m_{0}=n>m_{1}>\ldots>m_{t}$.

We obtain the digraph $\Theta_{j}^{-1}(T)$ from $T$ via the following procedure.

\section{Procedure for computing $\Theta_{j}^{-1}(T)$ :}

(1) First we declare that any edge $e$ of $T$ which is not an edge of the path from $n$ to $j$ is an edge of $\Theta_{j}^{-1}(T)$.

(2) Next we remove all edges of the form $\left(m_{t}, j\right)$ or $\left(m_{i-1}, x_{i}\right)$ for $1 \leq i \leq t$.

Finally for each $i$ with $1 \leq i \leq t$, we consider the subpath $x_{i}, \ldots, m_{i}$.

(3) If $m_{i}=x_{i}$, create a directed loop $\left(m_{i}, m_{i}\right)$.

(4) If $m_{i} \in C_{s}$ for some $s$, but $x_{i} \notin C_{s}$, convert the subpath $x_{i}, \ldots, m_{i}$ into the directed cycle $x_{i}, \ldots, m_{i}, x_{i}$.

(5) If $x_{i}, m_{i} \in C_{s}$ for some $s$, but $x_{i} \neq m_{i}$, then convert the subpath $x_{i}, x_{i}^{\prime}, \ldots, m_{i}$ to the directed cycle $x_{i}^{\prime}, \ldots, m_{i}, x_{i}^{\prime}$ and the directed loop $\left(x_{i}, x_{i}\right)$.

Lemma 2.5 If $T \in \mathcal{T}_{[m] ; j}^{G}$, then $\Theta_{j}^{-1}(T) \in \mathcal{F}_{n}(G, \mathbf{F}, m)$.

Proof: $\quad$ Suppose that $T \in \mathcal{T}_{[m] ; j}^{G}$. It is clear from the definition of $\Theta_{j}^{-1}$ that $\Theta_{j}^{-1}(T)$ is the digraph of a function $f$ with domain $\{m+1, \ldots, n-1\}$ and codomain $[n]$. That is, there are no edges out of the roots $1, \ldots, m$ in $T$ and we do not create any new edges out of $1, \ldots, m$ in the process of creating $\Theta_{j}^{-1}(T)$ from $T$. We remove the edge out of $n$ in $T$ and we do not create an edge out of $n$ in $\Theta_{j}^{-1}(T)$. Finally, for every vertex $v \in[n]-\{1, \ldots, m\} \cup\{n\}$, there will be an edge out of $v \in \Theta_{j}^{-1}(T)$. 
We need to show that items (1) through (3) of definition $\mathcal{F}_{n}(G, \mathbf{F}, m)$ are satisfied. The edges of $T$ are, by definition, edges of $G$. Thus any edge $(i, f(i))$ of $\Theta_{j}^{-1}(T)$ that was an edge of $T$ satisfies condition (1). Thus we need only consider the edges of $\Theta_{j}^{-1}(T)$ that are not in $T$.

These edges are the result of our procedure applied to the path

$$
m_{0}, x_{1}, \ldots, m_{1}, x_{2}, \ldots m_{2}, \ldots, x_{t}, \ldots, m_{t}, j
$$

where we set $m_{0}=n$. Note that all of the $m_{i}$ must be summit vertices of $G$. That is, all vertices which follow $m_{i}$ in the path must be less than $m_{i}$ by definition. Thus the edge out of $m_{i}$ on the path must be a downward edge. But the only downward edges in $G$ start at summit vertices so that $m_{i}$ must be a summit vertex.

Next suppose that there is a loop $(y, y)$ in $\Theta_{j}^{-1}(T)$ created from some $y \in \mathcal{C}_{p}$ for some $p$. Suppose that this loop is created from a subpath of the form $x_{a}, x_{a}^{\prime}, \ldots m_{a}$ for some $1 \leq a \leq t$. Thus $m_{a} \in \mathcal{C}_{p}$ so that $p>1$ since $m_{a}$ is a summit vertex. Note that $m_{a-1}>x_{a}$ so that $\left(m_{a-1}, x_{a}\right)$ is a downward edge in $G$ and hence $x_{a}$ is a base vertex. There are now two cases. First it could be that $x_{a}=m_{a}$, in which case $y=m_{a}=x_{a}$ so that $y$ is both a summit and a base vertex. Otherwise, $x_{a} \neq m_{a}, x_{a}, m_{a} \in \mathcal{C}_{p}$ and $y=x_{a}$. But in that case, $\mathcal{C}_{p}$ contains both a summit and base vertex and hence it must be both a summit and base. Thus we have shown that if $(y, y) \in \Theta_{j}^{-1}(T)$, then $y \in \mathcal{C}_{p}$ for some $p \in I_{B} \cap I_{S}$. Next suppose that a second such loop in $\mathcal{C}_{p}$ is created from a subpath $x_{b}, x_{b}^{\prime}, \ldots m_{b}$ where $a<b$. Then once again we can conclude that $x_{b}, m_{b} \in \mathcal{C}_{p}$. But this implies that $m_{b-1} \in \mathcal{C}_{e}$ where $e>p$ since $\left(m_{b-1}, x_{b}\right)$ is a downward edge. Thus, $m_{b-1}>m_{a}$, contradicting $a \leq b-1$ and the fact that $m_{0}>m_{1}>\ldots>m_{t}$. Thus we have shown that for any loop $(y, y)$ in $\Theta(T)$, there is a $p \in I_{B} \cap I_{S}$ such that $y \in \mathcal{C}_{p}$ and $(y, y)$ is the only loop that involves an element of $\mathcal{C}_{p}$. Thus conditions (2) and (3) of the definition of $\mathcal{F}_{n}(G, \mathbf{F}, q)$ are satisfied. Finally we observe that the argument just given shows that the element $y$ of the loop $(y, y)$ lies in the leftmost (as defined in the proof of Theorem 2.4) occurrence of a subpath of the form $x_{i}, x_{i}^{\prime}, \ldots m_{i}$ for which $m_{i} \in \mathcal{C}_{p}$. Thus our construction of $\Theta_{j}^{-1}$ is consistent with condition (d) of the proof of Theorem 2.4.

Finally, we must show that all nonloop edges of $\Theta_{j}^{-1}(T)$ that do not belong to $T$ must also belong to $E$. Such an edge can only arise from the path $x_{i}, \ldots, m_{i}$ where $x_{i} \neq m_{i}$. Since $m_{i}$ is the largest element on the path from $m_{i-1}$ to $j$, we know that $x_{i}<m_{i}$. There are now two cases. First it could be that this edge is of the form $\left(m_{i}, x_{i}\right)$ that arises from step (4) of our procedure defining $\Theta_{j}^{-1}$. Note that since $\left(m_{i-1}, x_{i}\right)$ is a downward edge of $G, x_{i}$ is a base vertex. But in case (4), $x_{i} \in C_{s}$ and $m_{i} \in C_{p}$ for some $s \neq p$ so that it must be the case that $s<p$ since $m_{i}>x_{i}$. Thus $\mathcal{C}_{s}$ is base which lies below the summit $\mathcal{C}_{p}$ and hence our definition of $G$ ensures that $\left(m_{i}, x_{i}\right)$ is an edge of $G$. The other case is where that path is of the form $x_{i}, x_{i}^{\prime}, \ldots, m_{i}$ where $x_{i}, m_{i} \in \mathcal{C}_{p}$ for some $p$ and the new edge is of the form $\left(m_{i}, x_{i}^{\prime}\right)$ that arises from step (5) of our procedure to define $\Theta_{j}^{-1}$. Thus $\left(x_{i}, x_{i}^{\prime}\right)$ an edge of $T$. It cannot be that $\left(x_{i}, x_{i}^{\prime}\right)$ is an upward edge since it would be the case that $x_{i}^{\prime}$ is in some $\mathcal{C}_{r}$ with $r>p$. But in that case $x_{i}^{\prime}>m_{i}$ since $m_{i} \in \mathcal{C}_{p}$ which would violate the fact that $m_{i}$ is the largest element on the path from $m_{i-1}$ to $j$. Thus it must be the case that $x_{i}>x_{i}^{\prime}$. Thus $\left(x_{i}, x_{i}^{\prime}\right)$ is a downward edge of $G$ so that $x_{i}^{\prime}$ is a 
base vertex. Since $x_{i} \in \mathcal{C}_{p}$, it must be that $x_{i}^{\prime} \in \mathcal{C}_{r}$ where $r<p$. Thus $\mathcal{C}_{r}$ is a base which lies below $\mathcal{C}_{p}$ which is a summit and hence $\left(m_{i}, x_{i}^{\prime}\right)$ is an edge of $G$. Thus, any nonloop edge added to $\Theta_{j}^{-1}(T)$ must be an edge of $G$ so that condition $(1)$ of $\mathcal{F}_{n}(G, \mathbf{F}, q)$ is also satisfied. This completes the proof of the lemma.

As noted in the proof just given, a loop $(y, y)$ with $y \in \mathcal{C}_{p}$ that appears in $\Theta_{j}^{-1}(T)$ occurs as the leftmost cycle of all cycles whose maximum element is also in $\mathcal{C}_{p}$. Thus, if we apply the map $\Theta_{j}$ to $\Theta_{j}^{-1}(T)$ then we will just reconstruct $T$. Thus $\Theta_{j}$ is a bijection from $\mathcal{F}_{n}(G, \mathbf{F}, q)$ to $\mathcal{T}_{[m] ; j}^{G}$ for each $1 \leq j \leq m$.

Next we consider the case were $N_{t-1}+1 \leq j \leq m$ so that $j \in \mathcal{C}_{t}$. Given a function $f^{*} \in \mathcal{F}_{n}^{*}(G, \mathbf{F}, m)$, the forest $\Theta_{j}^{*}\left(f^{*}\right)$ is created by exactly the same procedure that we used to create $\Theta_{j}(f)$ for $f \in \mathcal{F}_{n}(G, \mathbf{F}, m)$. To show that $\Theta_{j}^{*}\left(f^{*}\right)$ is always in $\mathcal{T}_{[m] ; j}^{G}$, one can use essentially the same argument that we used to show that $\Theta_{j}(f)$ is always in $\mathcal{T}_{[m] ; j}^{G}$ for an $f \in \mathcal{F}_{n}(G, \mathbf{F}, m)$ when $j \leq N_{t-1}$ with one exception. That is, consider the edge $\left(r_{c_{a}}\left(f^{*}\right), j\right)$. There are two possibilities. First it could be the $c_{a}$ is a one cycle so that $l_{c_{a}}\left(f^{*}\right)=r_{c_{a}}\left(f^{*}\right)$ and $r_{c_{a}}\left(f^{*}\right)$ is a fixed point of $f^{*}$. Then by definition of $\mathcal{F}_{n}^{*}(G, \mathbf{F}, m)$, it must be that $r_{c_{a}}\left(f^{*}\right) \in \mathcal{C}_{p}$ for $p>t$ and $r_{c_{a}}\left(f^{*}\right)$ is a summit vertex. Since $j$ is a base vertex in $\mathcal{C}_{t}$ and $p>t$, it follows that $\left(r_{c_{a}}\left(f^{*}\right), j\right)$ is an edge in $G$. The only other possibility is that $l_{c_{a}}\left(f^{*}\right) \neq r_{c_{a}}\left(f^{*}\right)$ in which case $\left(r_{c_{a}}\left(f^{*}\right), l_{c_{a}}\left(f^{*}\right)\right)$ is a downward edge. But then once again we can conclude that $r_{c_{a}}\left(f^{*}\right)$ is a summit vertex in some $\mathcal{C}_{p}$ for $p>t$. Thus once again in this case, $\left(r_{c_{a}}\left(f^{*}\right), j\right)$ is an edge in $G$ since $j$ is a base vertex in $\mathcal{C}_{t}$ and $p>t$.

Finally given a $T^{*} \in \mathcal{T}_{[m] ; j}^{G}$, we define $\left(\Theta_{j}^{*}\right)^{-1}\left(T^{*}\right)$ in exactly the same way that we defined $\Theta_{j}^{-1}(T)$ for $T \in \mathcal{T}_{[m] ; j^{\prime}}^{G}$ when $j^{\prime} \leq N_{t-1}$. The only thing that we have to check to prove that $\left(\Theta_{j}^{*}\right)^{-1}\left(T^{*}\right) \in \mathcal{F}_{n}^{*}(G, \mathbf{F}, m)$ is that $\left(\Theta_{j}^{*}\right)^{-1}\left(T^{*}\right)$ never has a fixed point in $\mathcal{C}_{t}$. That is, exactly the same argument will show that $\Theta_{j}^{*}\left(\left(\Theta_{j}^{*}\right)^{-1}\left(T^{*}\right)\right)=T^{*}$. It then follows from our ordering of the cycles that the only way that $f^{*}=\left(\Theta_{j}^{*}\right)^{-1}\left(T^{*}\right)$ could have a fixed point in $\mathcal{C}_{t}$ is if the rightmost cycle of $\left(\Theta_{j}^{*}\right)^{-1}\left(T^{*}\right)$ has its maximal element $r_{c_{a}}\left(F^{*}\right)$ in $\mathcal{C}_{p}$. But this is impossible since $j \in \mathcal{C}_{t}$ and $\left(r_{c_{a}}\left(f^{*}\right), j\right)$ is an edge in $G$.

We end this section with some general remarks about how to modify the proof of Theorem 2.4 to deal with root-directed spanning forests at other roots. For example, suppose that we want to find a formula for the number of root-directed spanning forests which are rooted at the $m$ largest vertices instead of the $m$ smallest vertices. One can easily modify the machinery that we developed to prove Theorem 2.4 to handle this case. That is, we need only make the following observation. Suppose that we replace each vertex label $i$ with the vertex label $n+1-i$. It is easy to see that we interchange ascent edges with descent edges and conversely. That is, suppose that $i \neq j$. Then an ascent edge $(i, j)$ with weight $p_{i} s_{j}$ will become a descent edge $(n+1-i, n+1-j)$ with weight $q_{n+1-i} t_{n+1-i}$. Similarly a descent edge $(i, j)$ with weight $q_{i} t_{j}$ becomes ascent edge $(n+1-i, n+1-j)$ with weight $p_{n+1-i} s_{n+1-i}$. In the case when $i=j$, we declared a loop $(i, i)$ as a descent edge with weight $q_{i} t_{i}$ which means that we want $(n+1-i, n+1-i)$ to be an ascent edge with weight $p_{n+1-i} s_{n+1-i}$. Thus the new weights of edges should be as follows. We define 
the weight of $(i, j), \bar{W}((i, j))$, by

$$
\bar{W}((i, j))=\left\{\begin{array}{l}
p_{i} s_{j} \text { if } i \leq j \\
q_{i} t_{j} \text { if } i>j
\end{array}\right.
$$

Similarly, we define the weight of any digraph $G=([n], E)$ by

$$
\bar{W}(G)=\prod_{(i, j) \in E} \bar{W}((i, j)) .
$$

If we do this, we can then get a formula for the sum of the weights $\bar{W}(F)$ of the rootdirected forests $F$ rooted at $n+1-1, \ldots, n+1-m$ by simply taking equation (4) and systematically replacing $q_{i}$ by $p_{n+1-i}, t_{i}$ by $s_{n+1-i}, p_{i}$ by $q_{n+1-i}$ and $s_{i}$ by $t_{n+1-i}$.

One can also develop a machinery to deal with root-directed forests rooted at an arbitrary set of roots. This was done in the case of a single root for the set of spanning trees of $K_{n}$ by Eğecioğlu and Remmel [4]. In that case, if one picks a root $i$ other than 1 or $n$, one must use a coarser set of weights than we used in Theorem 2.4. A similar situation holds for the case of root-directed forests of filter digraphs that are not rooted at the $m$ largest or the $m$ smallest vertices. However we shall not pursue these types of results in this paper.

\section{Examples}

Example 3.1. We use Theorem 2.4 to derive new results for the classical case of complete graphs. Consider the complete digraph $G=([n], E)$ where $E$ is the set of all pairs $(i, j)$, $i \neq j$. The graph $G$ corresponds in the obvious manner to $K_{n}$, the complete undirected graph on $n$. We take the composition $\mathbf{F}=\left(c_{1}, \ldots, c_{n}\right)$ of $n$ where $c_{i}=1$ for all $i$. The filtration $\left\{\mathcal{C}_{i} \mid i=1, \ldots, n\right\}$ associated with this composition of $n$ is the discrete partition. Note that $G=([n], E)$ is a filtered digraph with respect to $\mathbf{F}, I_{B}=\{1,2, \ldots, n-1\}$, and $I_{S}=\{2,3, \ldots, n\}$. Note that in this case, $\mathcal{F}_{n}(G, \mathbf{F}, m)=\mathcal{F}_{n}^{*}(G, \mathbf{F}, m)$ so that identity (5) of Theorem 2.4 becomes

$$
\begin{aligned}
\sum_{T \in \mathcal{T}_{[m]}^{G}} W(T) & =q_{n}\left(t_{1}+\cdots+t_{m}\right) \sum_{f \in \mathcal{F}_{n}(G, \mathbf{F}, m)} W(f) \\
& =q_{n}\left(t_{1}+\cdots+t_{m}\right) \prod_{j=m+1}^{n-1}\left(q_{j}\left(t_{1}+\ldots t_{j}\right)+p_{j}\left(s_{j+1}+\ldots+s_{n}\right)\right) .
\end{aligned}
$$

The set of root-directed spanning forests $T \in \mathcal{T}_{[\mathrm{m}]}^{G}$ with roots in $[m]$ corresponds in a natural way to the undirected spanning forests of $K_{n}$ with component trees rooted at the vertices $[m]$.

In the special case when $m=1,(8)$ becomes

$$
\sum_{T \in \mathcal{T}_{[1]}^{G}} W(T)=q_{n} t_{1} \prod_{j=2}^{n-1}\left(q_{j}\left(t_{1}+\ldots t_{j}\right)+p_{j}\left(s_{j+1}+\ldots+s_{n}\right)\right) .
$$


This formula easily specializes to the formula of Eğecioğlu and Remmel

$$
\sum_{T \in \mathcal{T}_{[1]}^{G}} \mathbf{W}(T)=x q^{n} y t^{1} \prod_{j=2}^{n-1}\left(x q^{j}\left(t^{1}+\ldots t^{j}\right)+y p^{j}\left(s^{j+1}+\ldots+s^{n}\right)\right)
$$

found in [4] where they used the weight $\mathbf{W}$ on edges $i \rightarrow j$ such that

$$
\mathbf{W}(i \rightarrow j)= \begin{cases}x q^{i} t^{j} & \text { if } i \geq j \\ y p^{i} s^{j} & \text { if } i<j\end{cases}
$$

by simply letting $q_{i}=x q^{i}, p_{i}=y p^{i}, s_{i}=s^{i}$ and $t_{i}=t^{i}$ for $i=1, \ldots, n$. We note however the proof given by Ĕgecioğlu and Remmel in [4] to prove (10) does prove (9) if one uses our weights.

The power of Theorem 2.4 is illustrated in this case by specializing the weight function $W$. Let $(A, B, N)$ be an ordered set partition of $\{m+1, \ldots, n-1\}$. We use the notation that $\chi($ statement $)=0$ if "statement" is false and $\chi($ statement $)=1$ if "statement" is true. For an edge $(i, j)$ of the graph $G$, define $\widetilde{W}((i, j))=p_{i} s_{j} \chi(i<j)$ if $i \in A$, $\widetilde{W}((i, j))=q_{i} t_{j} \chi(i \geq j)$ if $i \in D$, and $\widetilde{W}((i, j))=W((i, j))$ if $i \in N$ (see equation (1)). For a digraph $H=\left([n], E_{H}\right)$, let

$$
\widetilde{W}(H)=\prod_{(i, j) \in E_{H}} \widetilde{W}((i, j)) .
$$

Let $T$ be a root-directed spanning forest of $G$ with roots $1, \ldots m$. Note that $\widetilde{W}(T)$ is nonzero if and only if

for every vertex $i \in A, i$ is the first vertex of a directed edge $(i, j) \in E_{T}$ only if $i<j$ so that $(i, j)$ must be an "ascent" edge if $i \in A$ and

for every vertex $i \in D, i$ is the first vertex of a directed edge $(i, j) \in E_{T}$ only if $i \geq j$ so that $(i, j)$ must be a "descent edge" if $i \in D$.

Let $\mathcal{T}_{[m]}^{G}(A, D)$ denote the set of spanning forests of $T$ for which $\widetilde{W}(T)$ is nonzero. Equation (5) of Theorem 2.4 now can be used to express $\sum_{T \in \mathcal{T}_{[m]}^{G}(A, D)} \widetilde{W}(T)$ as

$q_{n}\left(t_{1}+\cdots+t_{m}\right) \prod_{i \in A} p_{i}\left(s_{i+1}+\cdots+s_{n}\right) \prod_{i \in D} q_{i}\left(t_{1}+\cdots+t_{i}\right) \prod_{i \in N}\left[q_{i}\left(t_{1}+\cdots+t_{i}\right)+p_{i}\left(s_{i+1}+\cdots+s_{n}\right)\right]$.

We now specialize further the weights by setting $p_{i}=q_{i}=s_{i}=t_{i}=q^{i}$. With this specialization,

$$
\sum_{T \in \mathcal{T}_{[m]}^{G}(A, D)} \widetilde{W}(T)=\sum_{T \in \mathcal{T}_{[m]}^{G}(A, D)} q^{\delta_{T}} \text { where } \delta_{T}=\sum_{i \in[n]} i \operatorname{deg}_{T}(i) .
$$


In this identity, $\operatorname{deg}_{T}(i)$ denotes the degree of the vertex $i$ in the tree $T$. Substituting the specialized weights into equation (8) and using the standard notation $[k]_{q}=1+q+\cdots+$ $q^{k-1}$ gives

$$
\sum_{T \in \mathcal{T}_{[m]}^{G}(A, D)} q^{\delta_{T}}=q^{\left(\left(\begin{array}{c}
n+2 \\
2
\end{array}\right)-\left(\begin{array}{c}
m+2 \\
2
\end{array}\right)\right)}[m]_{q}[n]_{q}^{|N|} \prod_{i \in A} q^{i}[n-i]_{q} \prod_{i \in D}[i]_{q} .
$$

Equation (14) is a new result for the complete graph that gives a specific formula for the $q$-generating function of a degree-weighted vertex-ranking statistic for spanning forests of $K_{n}$, with restricted ascents and descents, and with specified roots for the component trees of the forests.

Consider equation (14) in the case $A=D=\emptyset$. In that case, $N=\{m+1, \ldots, n-1\}$ and $T \in \mathcal{T}_{[m]}^{G}(A, D)=T \in \mathcal{T}_{[m]}^{G}$. We obtain,

$$
\sum_{T \in \mathcal{T}_{[m]}^{G}} q^{\delta_{T}}=q^{\left(\left(\begin{array}{c}
n+2 \\
2
\end{array}\right)-\left(\begin{array}{c}
m+2 \\
2
\end{array}\right)\right)}[m]_{q}[n]_{q}^{n-m-1} .
$$

In the case $m=1$ (a single root) we obtain

$$
\sum_{T \in \mathcal{T}_{1}^{G}} q^{\delta_{T}}=q^{\left(\left(\begin{array}{c}
n+2 \\
2
\end{array}\right)-3\right)}[n]_{q}^{n-2}
$$

Finally, if we take $q=1$, we obtain the classical formula for the number of Cayley trees.

$$
\left|\mathcal{T}_{1}^{G}\right|=n^{n-2} .
$$

This latter equation is the classical formula for the number of spanning trees of the complete graph. As a specific example, note that for $n=4$ and $m=1$, the right-hand side of equation (16) becomes $q^{12}[4]_{q}^{2}=q^{12}\left(1+q+q^{2}+q^{3}\right)^{2}$. This identity can easily be checked by listing the sixteen root-directed spanning trees for this case and comparing their weights with the sixteen terms in this expression. Note that the tree with largest weight 18 (i.e., 18 is the degree-weighted vertex-ranking statistic), corresponds to the term $q^{18}$. This tree has edges $(2,4),(3,4),(4,1)$. Likewise, the tree with smallest weight corresponds to the term $q^{12}$ and has edges $(2,1),(3,1),(4,1)$.

It is interesting to consider equation (14) in the case $A=N=\emptyset$. In that case, $D=$ $\{m+1, \ldots, n-1\}$. Now

$$
\sum_{T \in \mathcal{T}_{[m]}^{G}(\emptyset, D)} q^{\delta_{T}}=q^{\left(\left(\begin{array}{c}
n+2 \\
2
\end{array}\right)-\left(\begin{array}{c}
m+2 \\
2
\end{array}\right)\right)}[m]_{q} \prod_{i=m+1}^{n-1}[i]_{q} .
$$

In the case $m=1$, this equation becomes

$$
\sum_{T \in \mathcal{T}_{[m]}^{G}(\emptyset, D)} q^{\delta_{T}}=q^{\left(\left(\begin{array}{c}
n+2 \\
2
\end{array}\right)-3\right)}[n-1]_{q} ! .
$$


We use here the standard notation $\prod_{i=2}^{n-1}[i]_{q}=[n-1]_{q}$ !. As a specific example, take $n=4$ and $m=1$. Thus, $D=\{2,3\}$. The right-hand side of equation (16) becomes $q^{12}[2]_{q}[3]_{q}=q^{12}(1+q)\left(1+q+q^{2}\right)$. The six root-directed spanning trees with descent set $\{2,3\}$ have edge sets as follows:

$$
\begin{aligned}
& \{(2,1),(3,1),(4,1)\},\{(2,1),(3,2),(4,1)\},\{(2,1),(3,1),(4,3)\}, \\
& \{(2,1),(3,1),(4,2)\},\{(2,1),(3,2),(4,2)\},\{(2,1),(3,2),(4,3)\} .
\end{aligned}
$$

The analog of equation (19) in the case $D=N=\emptyset$ and $A=\{m+1, \ldots, n-1\}$ is easily derived.

Example 3.2. We next consider the case where $G=K_{n_{1}, \ldots, n_{k}}$ is the $n$-vertex multipartite graph with $k$ parts of size $n_{1}, \ldots, n_{k}$. Thus, $n=n_{1}+\cdots+n_{k}$. In this case, Onodera [8] showed that the number of spanning trees of $K_{n_{1}, \ldots, n_{k}}$ is $n^{k-2} \prod_{i=1}^{k}\left(n-k_{i}\right)^{k_{i}-1}$.

We start with the case $k=2$. In the context of this paper, the composition is $\mathbf{F}=\left(n_{1}, n_{2}\right)$. The filtration is $\left\{\mathcal{C}_{i} \mid i=1,2\right\}$ where $\mathcal{C}_{1}=\left\{1,2, \ldots, n_{1}\right\}$ and $\mathcal{C}_{2}=\left\{n_{1}+1, n_{1}+2, \ldots, n\right\}$. $\mathcal{C}_{1}$ is a base and $\mathcal{C}_{2}$ is a summit. We assume that $1 \leq m \leq n_{1}$ so that equation (5) becomes

$$
\begin{aligned}
& \sum_{T \in \mathcal{T}_{[m]}^{G}} W(T)=q_{n}\left(t_{1}+\cdots+t_{m}\right) \sum_{f \in \mathcal{F}_{n}(G, \mathbf{F}, m)} W(f) \\
= & q_{n}\left(t_{1}+\cdots+t_{m}\right) \prod_{j=m+1}^{n_{1}} p_{j}\left(s_{n_{1}+1}+\ldots+s_{n}\right) \prod_{j=1}^{n_{2}-1} q_{n_{1}+j}\left(t_{1}+\ldots+t_{n_{1}}\right) .
\end{aligned}
$$

The set of root-directed spanning forests $T \in \mathcal{T}_{[m]}^{G}$, with roots $[m]$, corresponds in a natural way to the undirected spanning forests of $K_{n_{1}, n_{2}}$, with component trees rooted at the vertices $[m]$. As in the previous example, we specialize further the weights by setting $p_{i}=q_{i}=s_{i}=t_{i}=q^{i}$. This substitution, then leads to the following calculation:

$$
\begin{aligned}
& q^{n+1}[m]_{q} \prod_{j=m+1}^{n_{1}} q^{j+n_{1}+1}\left[n_{2}\right]_{q} \prod_{j=1}^{n_{2}-1} q^{n_{1}+j+1}\left[n_{1}\right]_{q} \\
= & q^{n+1}[m]_{q} q^{n_{1}\left(n_{1}-m\right)}\left[n_{2}\right]_{q}^{n_{1}-m}\left[n_{1}\right]_{q}^{n_{2}-1} \prod_{j=m+1}^{n_{1}} q^{j+1} \prod_{j=n_{1}+1}^{n_{2}-1} q^{n_{1}+j+1} \\
= & q^{n+1}[m]_{q} q^{n_{1}\left(n_{1}-m\right)}\left[n_{2}\right]_{q}^{n_{1}-m}\left[n_{1}\right]_{q}^{n_{2}-1} \prod_{j=m+1}^{n-1} q^{j+1} \\
= & {[m]_{q} q^{n_{1}\left(n_{1}-m\right)}\left[n_{2}\right]_{q}^{n_{1}-m}\left[n_{1}\right]_{q}^{n_{2}-1} \prod_{j=m+1}^{n} q^{j+1} } \\
= & {[m]_{q} q^{n_{1}\left(n_{1}-m\right)}\left[n_{2}\right]_{q}^{n_{1}-m}\left[n_{1}\right]_{q}^{n_{2}-1} q\left(\left(\begin{array}{c}
n+2 \\
2
\end{array}\right)-\left(\begin{array}{c}
m+2 \\
2
\end{array}\right)\right) }
\end{aligned}
$$

Analogous to equation (15) of the previous example, we obtain

$$
\sum_{T \in \mathcal{T}_{[m]}^{G}} q^{\delta_{T}}=q^{\left(\left(\begin{array}{c}
n+2 \\
2
\end{array}\right)-\left(\begin{array}{c}
m+2 \\
2
\end{array}\right)\right)}[m]_{q} q^{n_{1}\left(n_{1}-m\right)}\left[n_{1}\right]_{q}^{n_{2}-1}\left[n_{2}\right]_{q}^{n_{1}-m} .
$$


As a trivial special case of this equation, we can easily verify the case $n=4, n_{1}=n_{2}=2$. If $m=1$, we get $q^{14}(1+q)^{2}=q^{14}+2 q^{15}+q^{16}$ for the right-hand side of equation (21). The tree with degree-weighted vertex-ranking statistic 14 has edge set $\{(3,1),(4,1),(2,3)\}$, the two trees with statistic 15 have edge sets $\{(3,1),(4,1),(2,4)\}$ and $\{(3,1),(2,3),(4,2)\}$, and the tree with statistic 16 has edge set $\{(3,2),(4,1),(2,4)\}$. If, on the other hand, we take $m=2$, we obtain $q^{9}(1+q)^{2}=q^{9}+2 q^{10}+q^{11}$ for the right-hand side of equation (21). The tree with statistic 9 has edge set $\{(3,1),(4,1)\}$ (the tree rooted at 2 has no edges). The two trees with statistic 10 have edge sets $\{(3,2),(4,1)\}$ and $\{(3,1),(4,2)\}$. The tree with statistic 11 has edge set $\{(3,2),(4,2)\}$.

We now consider the more difficult case of $k>2$. In this case we will need the full power of Theorem 2.4 in that we will need to consider functions whose associated digraphs have loops. In particular, $K_{n_{1}, \ldots, n_{k}}$ is the $n$-vertex multipartite graph with $k>2$ parts of size $n_{1}, \ldots, n_{k}$. Thus, $n=n_{1}+\cdots+n_{k}$ and the composition is $\mathbf{F}=\left(n_{1}, \ldots n_{k}\right)$. Again, we denote the partial sum $n_{1}+\cdots+n_{j}$ by $N_{j}$ and let $N_{0}=0$. The filtration in this case is $\left\{\mathcal{C}_{i} \mid i=1, \ldots k\right\}$ where

$\mathcal{C}_{1}=\left\{1,2, \ldots, N_{1}\right\}, \mathcal{C}_{2}=\left\{N_{1}+1, N_{1}+2, \ldots, N_{2}\right\}, \ldots, \mathcal{C}_{k}=\left\{N_{k-1}+1, N_{k-1}+2, \ldots, N_{k}\right\}$.

$\mathcal{C}_{1}, \ldots, \mathcal{C}_{k-1}$ are bases and $\mathcal{C}_{2}, \ldots, \mathcal{C}_{k}$ are summits. Take $1 \leq m \leq N_{k-1}$ and assume that $m \in \mathcal{C}_{t}$. Identity (5) of Theorem 2.4 then becomes

$$
\left[q_{n}\left(t_{1}+\cdots+t_{N_{t-1}}\right) \tilde{B}_{t} \prod_{j=t+1}^{k} \tilde{A}_{j}\right]+\left[q_{n}\left(t_{N_{t-1}+1} \cdots+t_{m}\right) \tilde{D}_{t} \prod_{j=t+1}^{k} \tilde{A}_{j}\right]=\sum_{T \in \mathcal{T}_{[m]}^{G}} W(T) .
$$

Here for $t<j<k$,

$$
\begin{gathered}
\tilde{A}_{j}=\prod_{i=N_{j-1}+1}^{N_{j}}\left(p_{i}\left(s_{N_{j}+1}+\cdots+s_{n}\right)+q_{i}\left(t_{1}+\cdots+t_{N_{j-1}}\right)\right) \\
+\sum_{r=N_{j-1}+1}^{N_{j}} q_{r} t_{r} \prod_{\substack{i=N_{j-1}+1 \\
i \neq r}}^{N_{j}}\left(p_{i}\left(s_{N_{j}+1}+\cdots+s_{n}\right)+q_{i}\left(t_{1}+\cdots+t_{N_{j-1}}\right)\right) .
\end{gathered}
$$

The summation in the above expression takes into account the possible loops in the digraphs corresponding to the functions $f \in \mathcal{F}_{n}(G, \mathbf{F}, m)$. Similarly,

$$
\begin{gathered}
\tilde{B}_{t}=\prod_{i=m+1}^{N_{t}}\left(p_{i}\left(s_{N_{t}+1}+\cdots+s_{n}\right)+q_{i}\left(t_{1}+\cdots+t_{N_{t-1}}\right)\right) \\
+\sum_{r=m+1}^{N_{t}} q_{r} t_{r} \prod_{\substack{i=m+1 \\
i \neq r}}^{N_{t}}\left(p_{i}\left(s_{N_{t}+1}+\cdots+s_{n}\right)+q_{i}\left(t_{1}+\cdots+t_{N_{t-1}}\right)\right)
\end{gathered}
$$


and

$$
\tilde{D}_{t}=\prod_{i=m+1}^{N_{t}}\left(p_{i}\left(s_{N_{t}+1}+\cdots+s_{n}\right)+q_{i}\left(t_{1}+\cdots+t_{N_{t-1}}\right)\right) .
$$

Note that if $t=1, \tilde{D}_{t}=\prod_{i=m+1}^{N_{t}} p_{i}\left(s_{N_{t}+1}+\cdots+s_{n}\right)$. Also, if $m=N_{t}$, then we take $\tilde{B}_{t}=\tilde{D}_{t}=1$. Finally, for $j=k$, we have

$$
\tilde{A}_{k}=\prod_{i=N_{k-1}+1}^{n-1} q_{i}\left(t_{1}+\cdots t_{N_{k-1}}\right) .
$$

As a simple check on equation (22), take $n=6, n_{1}=n_{2}=n_{3}=2$ and $m=2$ so that $t=1$ and $N_{t-1}=0$. We set all variables equal to 1 . Note that, by our convention, $t_{1}+\cdots+t_{N_{t-1}}=0$ in this case. Since $m=N_{1}, \tilde{D}_{1}=1$ by convention. Thus we must compute the value of $q_{6}\left(t_{1}+t_{2}\right) \tilde{D}_{1} \tilde{A}_{2} \tilde{A}_{3}$ when all the variables are set equal to 1 . It is easy to see that under this substitution, $\tilde{A}_{2}$ becomes 24 and $\tilde{A}_{3}$ becomes 4 so that the final result is $2 \times 24 \times 4=192$. Thus there are 192 root-directed spanning forests for this 3 -partite graph with roots 1 and 2 . This can be checked directly from the problem definition (with a bit of work). Similarly, suppose that we take $n=6, n_{1}=n_{2}=n_{3}=2$ and $m=3$ so that $t=3$ and $N_{t-1}=2$. Then we must compute $q_{6}\left(t_{1}+t_{2}\right) \tilde{B}_{2} \tilde{A}_{3}+q_{6} t_{3} \tilde{D}_{2} \tilde{A}_{3}$ when all the variables are set equal to 1 . It is easy to check that under this substitution $\tilde{B}_{2}$ becomes 5 and $\tilde{D}_{2}$ becomes 4 so that there are $2 \times 5 \times 4+4 \times 4=56$ root-directed spanning forests for this 3-partite graph with roots 1,2 and 3 .

We note that in the special case when $m=1$, Eğecioğlu and Remmel in [6] had a specialization of (22) for $G=K_{n_{1}, \ldots, n_{k}}$. That is, they again used the weight $\mathbf{W}$ on edges $i \rightarrow j$ such that

$$
\mathbf{W}(i \rightarrow j)= \begin{cases}x q^{i} t^{j} & \text { if } i \geq j \\ y p^{i} s^{j} & \text { if } i<j\end{cases}
$$

and proved that

$$
\sum_{T \in \mathcal{T}_{[1]}^{G}} \mathbf{W}(T)=x q^{n} y t^{1} G F\left(F_{1}\right) \cdots G\left(F_{k}\right)
$$

where

$$
\begin{gathered}
G F\left(F_{1}\right)=\prod_{i=2}^{N_{1}} y p^{i}\left(s^{1+N_{1}}+\cdots+s^{n}\right), \\
G F\left(F_{k}\right)=\prod_{i=1+N_{k-1}}^{n-1} x q^{i}\left(t+\cdots+t^{N_{k-1}}\right)
\end{gathered}
$$


and for $1<t<k$,

$$
\begin{aligned}
& G F\left(F_{t}\right)= \\
& \prod_{i=1+N_{t-1}}^{N_{t}}\left(y p^{i}\left(s^{1+N_{t}}+\cdots+s^{n}\right)+\left(x q^{i}\left(t+\cdots+t^{N_{t-1}}\right)\right)\right. \\
& +\sum_{i=1+N_{t-1}}^{N_{t}} x q^{i} t^{i} \prod_{\substack{j=1+N_{t-1} \\
j \neq i}}^{N_{t}}\left(y p^{i}\left(s^{1+N_{t}}+\cdots+s^{n}\right)+\left(x q^{i}\left(t+\cdots+t^{N_{t-1}}\right)\right) .\right.
\end{aligned}
$$

We now turn to the specialization of equation (22), analogous to equation (14) of the previous example, that gives us the generating function for the degree-weighted vertexranking statistics associated with spanning forests of the multipartite graph $G=K_{n_{1}, \ldots, n_{k}}$. We set all variables subscripted by $i$ in equation (22) equal to $q^{i}$. To simplify the notation, we use the same symbols as used previously for equation (22) (i.e., $\tilde{A}_{j}$, $\tilde{B}_{t}$, etc.). We obtain from equation (22),

$$
\left[q^{n+1}\left[N_{t-1}\right]_{q} \tilde{B}_{t} \prod_{j=t+1}^{k} \tilde{A}_{j}\right]+\left[q^{n+1} q^{N_{t-1}}\left[m-N_{t-1}\right]_{q} \tilde{D}_{t} \prod_{j=t+1}^{k} \tilde{A}_{j}\right]=\sum_{T \in \mathcal{T}_{[m]}^{G}} q^{\delta_{T}}
$$

Now we have, for $t<j<k$,

$$
\begin{aligned}
& \tilde{A}_{j}=\left(\prod_{i=N_{j-1}+1}^{N_{j}} q^{i+1}\right) \times \\
& \left(\prod_{i=N_{j-1}+1}^{N_{j}}\left(q^{N_{j}}\left[n-N_{j}\right]_{q}+\left[N_{j-1}\right]_{q}\right)\right. \\
& \left.+\sum_{r=N_{j-1}+1}^{N_{j}} q^{r-1} \prod_{\substack{i=N_{j-1}+1 \\
i \neq r}}^{N_{j}}\left(q^{N_{j}}\left[n-N_{j}\right]_{q}+\left[N_{j-1}\right]_{q}\right)\right) .
\end{aligned}
$$

Rewriting the inner products as powers and factoring them from the sum gives

$$
\begin{aligned}
& \tilde{A}_{j}=\left(\prod_{i=N_{j-1}+1}^{N_{j}} q^{i+1}\right) \times \\
& \left(\left(q^{N_{j}}\left[n-N_{j}\right]_{q}+\left[N_{j-1}\right]_{q}\right)^{N_{j}-N_{j-1}}\right. \\
& \left.+\left(q^{N_{j}}\left[n-N_{j}\right]_{q}+\left[N_{j-1}\right]_{q}\right)^{N_{j}-N_{j-1}-1} q^{N_{j-1}}\left[N_{j}-N_{j-1}\right]_{q}\right) .
\end{aligned}
$$

Here the sum $\sum_{r=N_{j-1}+1}^{N_{j}} q^{r-1}$ has been rewritten as $q^{N_{j-1}}\left[N_{j}-N_{j-1}\right]_{q}$. Next we can factor out $\left(q^{N_{j}}\left[n-N_{j}\right]_{q}+\left[N_{j-1}\right]_{q}\right)^{N_{j}-N_{j-1}-1}$ and observe that $n_{j}=N_{j}-N_{j-1}$ and $[n]_{q}=$ 
$q^{N_{j}}\left[n-N_{j}\right]_{q}+\left[N_{j-1}\right]_{q}+q^{N_{j-1}}\left[N_{j}-N_{j-1}\right]_{q}$ to obtain

$$
\tilde{A}_{j}=[n]_{q}\left(\prod_{i=N_{j-1}+1}^{N_{j}} q^{i+1}\right)\left(q^{N_{j}}\left[n-N_{j}\right]_{q}+\left[N_{j-1}\right]_{q}\right)^{n_{j}-1}
$$

An almost identical computation yields

$$
\tilde{B}_{t}=\left(\prod_{i=m+1}^{N_{t}} q^{i+1}\right)\left(q^{N_{t}}\left[n-N_{t}\right]_{q}+\left[N_{t-1}\right]_{q}\right)^{N_{t}-m-1}\left(q^{N_{t}}\left[n-N_{t}\right]_{q}+\left[N_{t-1}\right]_{q}+q^{m}\left[N_{t}-m\right]_{q}\right) .
$$

Similarly,

$$
\tilde{D}_{t}=\left(\prod_{i=m+1}^{N_{t}} q^{i+1}\right)\left(q^{N_{t}}\left[n-N_{t}\right]_{q}+\left[N_{t-1}\right]_{q}\right)^{N_{t}-m}
$$

and

$$
\tilde{A}_{k}=\left(\prod_{i=N_{k-1}+1}^{n-1} q^{i+1}\right)\left[n-n_{k}\right]_{q}^{n_{k}-1}
$$

We now substitute the above identities into equation (24) and collect together the $k-t-1$ factors of $[n]_{q}$. We also combine the initial product expressions in the above identities which, together with the factor $q^{n+1}$, give

$$
\prod_{i=m+1}^{n} q^{i+1}=q^{\left(\begin{array}{c}
n+2 \\
2
\end{array}\right)-\left(\begin{array}{c}
m+2 \\
2
\end{array}\right)}
$$

The resulting identity is the " $q$-version" of equation $(22)\left(W(T)=q^{\delta_{T}}\right.$ is the $q$-weight):

$$
[n]_{q}^{k-t-1} q^{\left(\begin{array}{c}
n+2 \\
2
\end{array}\right)-\left(\begin{array}{c}
m+2 \\
2
\end{array}\right)}\left(\left[N_{t-1}\right]_{q} B_{t}+q^{N_{t-1}}\left[m-N_{t-1}\right]_{q} D_{t}\right) \prod_{j=t+1}^{k} A_{j}=\sum_{T \in \mathcal{T}_{[m]}^{G}} q^{\delta_{T}}
$$

where,

$$
\begin{aligned}
& B_{t}=\left(q^{N_{t}}\left[n-N_{t}\right]_{q}+[\right.\left.\left.N_{t-1}\right]_{q}\right)^{N_{t}-m-1}\left(q^{N_{t}}\left[n-N_{t}\right]_{q}+\left[N_{t-1}\right]_{q}+q^{m}\left[N_{t}-m\right]_{q}\right) \\
&=\left([n]_{q}-q^{N_{t-1}}\left[n_{t}\right]_{q}\right)^{N_{t}-m-1}\left([n]_{q}-q^{N_{t-1}}\left[m-N_{t-1}\right]_{q}\right), \\
& D_{t}=\left(q^{N_{t}}\left[n-N_{t}\right]_{q}+\left[N_{t-1}\right]_{q}\right)^{N_{t}-m} \\
&=\left([n]_{q}-q^{N_{t-1}}\left[n_{t}\right]_{q}\right)^{N_{t}-m}, \\
& A_{j}=\left(q^{N_{j}}\left[n-N_{j}\right]_{q}+\left[N_{j-1}\right]_{q}\right)^{n_{j}-1} \\
&=\left([n]_{q}-q^{N_{j-1}}\left[n_{j}\right]_{q}\right)^{n_{j}-1}
\end{aligned}
$$


for $t<j<k$, and

$$
A_{k}=\left[n-n_{k}\right]_{q}^{n_{k}-1}
$$

We consider some special cases. If $q=1$, then equation (25) gives the cardinality $\left|\mathcal{T}_{[m]}^{G}\right|$. In this case, $A_{j}=\left(n-n_{j}\right)^{n_{j}-1}, t<j \leq k . \quad B_{t}=\left(n-n_{t}\right)^{N_{t}-m-1}\left(n+N_{t-1}-m\right)$ and $D_{t}=\left(n-n_{t}\right)^{N_{t}-m}$. We obtain,

$$
n^{k-t-1}\left(N_{t-1}\left(n-n_{t}\right)^{N_{t}-m-1}\left(n+N_{t-1}-m\right)+\left(m-N_{t-1}\right)\left(n-n_{t}\right)^{N_{t}-m}\right) \prod_{j=t+1}^{k}\left(n-n_{j}\right)^{n_{j}-1}
$$

for $\left|\mathcal{T}_{[m]}^{G}\right|$. Note that if $t=1, N_{t-1}=N_{0}=0$ and thus we obtain

$$
n^{k-2}\left(m\left(n-n_{t}\right)^{n_{1}-m}\right) \prod_{j=2}^{k}\left(n-n_{j}\right)^{n_{j}-1}=\left|T \in \mathcal{T}_{[m]}^{G}\right| .
$$

If $m=1$ this identity becomes the formula of Onodera [8]:

$$
n^{k-2} \prod_{j=1}^{k}\left(n-n_{j}\right)^{n_{j}-1}=\left|\mathcal{T}_{1}^{G}\right|
$$

To take some simple numerical examples, consider the case considered above where $n=6$, $n_{1}=n_{2}=n_{3}=2, t=1$ and $m=2$ (hence $N_{t-1}=0$ ). We set all variables equal to 1 in the expression

$$
n^{k-2}\left(m\left(n-n_{t}\right)^{n_{1}-m}\right) \prod_{j=2}^{k}\left(n-n_{j}\right)^{n_{j}-1}=\left|\mathcal{T}_{[m]}^{G}\right|
$$

obtaining $6 \times 2 \times 4^{0} \times 4 \times 4=192$ as previously found. To take a more complex example, consider the case where $n=6, n_{1}=n_{2}=n_{3}=2, t=2$ and $m=3$. The left-hand side of equation (22) becomes

$$
q^{18}\left(1+q+q^{2}+q^{3}\right)\left((1+q)\left(\left(1+q^{4}\right)(1+q)+q^{3}\right)+q^{2}\left(1+q^{4}\right)(1+q)\right) .
$$

Expanding this expression, we obtain

$$
q^{18}+3 q^{19}+5 q^{20}+7 q^{21}+8 q^{22}+8 q^{23}+8 q^{24}+7 q^{25}+5 q^{26}+3 q^{27}+q^{28}
$$

as the degree-weighted vertex-ranking generating function for the spanning forests of $G$ with roots 1,2 , and 3 . This can be checked without too much difficulty directly from the problem definition.

Finally, we note that equation (25) with $k=2$ and $t=1$ reduces to equation (21). Substituting these values for $k$ and $t$ into equation (25) into the expressions $B_{1}, D_{t}, A_{j}$, and $A_{k}$ associated with equation (25) gives $[n]_{q}^{2-1-1}=1,\left[N_{0}\right]_{q} B_{1}=0$, and

$$
\begin{aligned}
D_{1} & =\left(q^{n_{1}}\left[n-n_{1}\right]_{q}\right)^{n_{1}-m} \\
& =q^{n_{1}\left(n_{1}-m\right)}\left[n-n_{1}\right]_{q}^{n_{1}-m}
\end{aligned}
$$


and hence

$$
q^{N_{0}}\left[m-N_{0}\right]_{q} D_{1}=[m]_{q} D_{1}=[m]_{q} q^{n_{1}\left(n_{1}-m\right)}\left[n-n_{1}\right]_{q}^{n_{1}-m} .
$$

There are no values $j$ such that $1=t<j<k=2$ in this case. For $k=2, A_{2}=$ $\left[n-n_{2}\right]_{q}^{n_{2}-1}$. Thus, the left-hand side of equation (25) becomes

$$
q^{\left(\begin{array}{c}
n+2 \\
2
\end{array}\right)-\left(\begin{array}{c}
m+2 \\
2
\end{array}\right)}[m]_{q} D_{1} A_{2}=q^{\left(\begin{array}{c}
n+2 \\
2
\end{array}\right)-\left(\begin{array}{c}
m+2 \\
2
\end{array}\right)}[m]_{q} q^{n_{1}\left(n_{1}-m\right)}\left[n-n_{1}\right]_{q}^{n_{1}-m}\left[n-n_{2}\right]_{q}^{n_{2}-1} .
$$

Thus, equation (24) becomes

$$
q^{\left(\left(\begin{array}{c}
n+2 \\
2
\end{array}\right)-\left(\begin{array}{c}
m+2 \\
2
\end{array}\right)\right)}[m]_{q} q^{n_{1}\left(n_{1}-m\right)}\left[n_{1}\right]_{q}^{n_{2}-1}\left[n_{2}\right]_{q}^{n_{1}-m}=\sum_{T \in \mathcal{T}_{[m]}^{G}} q^{\delta_{T}} .
$$

This latter equation is exactly equation (21).

Example 3.3. In this example, we consider a directed graph $G=C_{n_{1}, \ldots, n_{k}}$ which is not the digraph corresponding to an undirected graph (as were examples (3.1) and (3.2)). This graph is a multipartite cyclic digraph defined by taking the composition of $n$ to be $\mathbf{F}=\left(n_{1}, \ldots, n_{k}\right)$. Thus, the filtration is the partition $\mathcal{C}_{i}=\left\{N_{i-1}+1, \ldots, N_{i}\right\}$, with $N_{0}=0$ and $N_{i}=n_{1}+\cdots+n_{i}, i=1, \ldots, k$. There is one base, $\mathcal{C}_{1}$, and one summit, $\mathcal{C}_{k}$. All directed edges that connect a vertex in $\mathcal{C}_{i}$ to a vertex in $\mathcal{C}_{i+1}$ are present for $i=1, \ldots, k-1$. As required by our framework (Theorem 2.4), all directed edges that connect a summit vertex in $\mathcal{C}_{k}$ to a base vertex in $\mathcal{C}_{1}$ are also present. Thus in this case, the $t$ as in the statement of Theorem 2.4 is just 1 . Thus all roots $\{1, \ldots, m\}$ of forests in $\mathcal{T}_{[m]}^{G}$ will belong to $\mathcal{C}_{1}$ since all such roots belong to a base and $\mathcal{C}_{1}$ is the only base.

As with equations (8) and (22), we start with identity (5) of Theorem 2.4:

$$
\begin{aligned}
& \sum_{T \in \mathcal{T}_{[m]}^{G}} W(T)= \\
& q_{n}\left(t_{1}+\cdots+t_{N_{t-1}}\right) \sum_{f \in \mathcal{F}_{n}(G, \mathbf{F}, m)} W(f)+q_{n}\left(t_{N_{t-1}+1}+\cdots+t_{m}\right) \sum_{f \in \mathcal{F}_{n}^{*}(G, \mathbf{F}, m)} W(f) .
\end{aligned}
$$

In this case, $N_{t-1}=0$ so we obtain the simplification

$$
q_{n}\left(t_{1}+\cdots+t_{m}\right) \sum_{f \in \mathcal{F}_{n}^{*}(G, \mathbf{F}, m)} W(f)=\sum_{T \in \mathcal{T}_{[m]}^{G}} W(T) .
$$

Translating the sum over $f \in \mathcal{F}_{n}^{*}(G, \mathbf{F}, m)$ directly into generating functions we obtain

$$
q_{n}\left(t_{1}+\cdots+t_{m}\right) \tilde{D}_{1} \prod_{j=2}^{k} \tilde{A}_{j}=\sum_{T \in \mathcal{T}_{[m]}^{G}} W(T) .
$$

Here for $t<j<k$,

$$
\tilde{A}_{j}=\prod_{i=N_{j-1}+1}^{N_{j}} p_{i}\left(s_{N_{j}+1}+\cdots+s_{N_{j+1}}\right) .
$$


The summation in the above expression does not take into account fixed points in functions $f \in \mathcal{F}_{n}(G, \mathbf{F}, m)$. No such fixed points can occur since any such point must belong to both a base and a summit and there are no blocks in the filtration that are both a base and a summit. In addition, we have

$$
\tilde{D}_{1}=\prod_{i=m+1}^{N_{1}} p_{i}\left(s_{N_{1}+1}+\cdots+s_{N_{2}}\right) \quad \text { and } \quad \tilde{A}_{k}=\prod_{i=N_{k-1}+1}^{n-1} q_{i}\left(t_{1}+\cdots t_{N_{1}}\right) .
$$

We now turn to the specialization of equation (31) that gives us the generating function for the degree-weighted vertex-ranking statistics associated with spanning forests of the multipartite cyclic graph $G=C_{n_{1}, \ldots, n_{k}}$. We set all variables subscripted by $i$ in equation (31) equal to $q^{i}$. As in the previous example, we shall use the same symbols as we used in connection with equation (31) (i.e., $\tilde{A}_{j}, \tilde{D}_{1}$ ). We obtain from equation (31), in a manner similar to the previous example,

$$
q^{n+1}[m]_{q} \tilde{D}_{1} \prod_{j=2}^{k} \tilde{A}_{j}=\sum_{T \in \mathcal{T}_{[m]}^{G}} q^{\delta_{T}}
$$

where

$$
\tilde{D}_{1}=\left(\prod_{i=m+1}^{n_{1}} q^{i+1}\right)\left(q^{n_{1}}\left[n_{2}\right]_{q}\right)^{n_{1}-m}
$$

for $1<j<k$

$$
\tilde{A}_{j}=\left(\prod_{i=N_{j-1}+1}^{N_{j}} q^{i+1}\right)\left(q^{N_{j}}\left[n_{j+1}\right]_{q}\right)^{n_{j}},
$$

and, finally,

$$
\tilde{A}_{k}=\left(\prod_{i=N_{k-1}+1}^{n-1} q^{i+1}\right)\left[n_{1}\right]_{q}^{n_{k}-1} .
$$

Combining the products in the above expressions, we obtain

$$
[m]_{q} q^{\left(\begin{array}{c}
n+2 \\
2
\end{array}\right)-\left(\begin{array}{c}
m+2 \\
2
\end{array}\right)} D_{1} \prod_{j=2}^{k} A_{j}=\sum_{T \in \mathcal{T}_{[m]}^{G}} q^{\delta_{T}}
$$

where

$$
D_{1}=\left(q^{n_{1}}\left[n_{2}\right]_{q}\right)^{n_{1}-m},
$$

for $1<j<k$,

$$
A_{j}=\left(q^{N_{j}}\left[n_{j+1}\right]_{q}\right)^{n_{j}},
$$

and

$$
A_{k}=\left[n_{1}\right]_{q}^{n_{k}-1} .
$$


We note that it is easily shown that for the case $k=2$, equation (32) reduces to equation (21) as it should.

As a simple check of equation (32), take $n=6, n_{1}=n_{2}=n_{3}=2$, and $m=1$. We obtain

$$
q^{25}\left(q^{2}(1+q)\right)\left(q^{4}(1+q) q^{4}(1+q)\right)(1+q) .
$$

Expanding, this becomes

$$
q^{35}+4 q^{36}+6 q^{37}+4 q^{38}+q^{39}
$$

These functions are easily listed directly from the problem definition. For example, the minimal weight root-directed spanning tree with $\delta_{T}=35$ has edge set

$$
\{(2,3),(3,5),(4,5),(5,1),(6,1)\}
$$

and the maximal weight spanning tree with $\delta_{T}=39$ has edge set

$$
\{(5,2),(2,4),(4,6),(3,6),(6,1)\} .
$$

Finally we end this section with a brief discussion of how one can generate additional examples of filtered digraphs by modifying and combining examples 3.2 and 3.3 in various ways.

First, we should note that Example 3.3 is the simplest case of a more general construction. That is, suppose that we start with any acylic directed graph $G=([k], E)$ such that vertex 1 is the only source in $G$, vertex $k$ is the only $\operatorname{sink}$ in $G$, and all vertices lie on a path from the source to the sink. Then we can create a filtered digraph as follows. Start with a composition $\mathbf{F}=\left(c_{1}, c_{2}, \ldots, c_{k}\right)$ of $n$ and let $\mathcal{C}_{1}, \ldots, \mathcal{C}_{k}$ be its corresponding set partition. Next we replace each vertex i by an empty graph $\mathcal{C}_{i}$. Then whenever there is an edge from $(i, j) \in E$, we create a directed edge from each vertex in $\mathcal{C}_{i}$ to each vertex in $\mathcal{C}_{j}$. We let $\mathcal{C}_{1}$ be the only base and let $\mathcal{C}_{k}$ be the only summit. Finally, we must add a directed edge from each summit vertex to each base vertex. Clearly, the graphs $C_{n_{1}, \ldots, n_{k}}$ arise from this construction by starting with a simple graph

$$
1 \rightarrow 2 \rightarrow \cdots \rightarrow k-1 \rightarrow k
$$

We can also combine Examples 3.2 and 3.3 in various ways. For example, suppose that we start with blocks of sizes $n_{1}, \ldots, n_{k}$ as in Example 3.2. Now instead of taking the empty graph in each of these blocks as we do to create $K_{n_{1}, \ldots, n_{k}}$, imagine that we put the structure of a multipartite cyclic digraph in each block. That is, suppose that in each block $\mathcal{C}_{i}=\left\{N_{i-1}+1, \ldots, N_{i}\right\}$, we put the structure of some $C_{p_{1}, \ldots, p_{l}}$ where $p_{1}+\cdots+p_{l}=n_{i}$. Then there are many possible ways to connect the blocks $\mathcal{C}_{1}, \ldots, \mathcal{C}_{k}$ to form a filtered digraph. Clearly we have to connect each vertex in a summit of a $C_{p_{1}, \ldots, p_{l}}$ making up a block $\mathcal{C}_{i}$ to all vertices in bases in a block $\mathcal{C}_{s}$ with $s<i$. However, we have many choices of how to connect the element of $\mathcal{C}_{i}$ to elements of $\mathcal{C}_{t}$ with $i<t$ and still conform to Definition 2.2. For example, we could have a directed edge from each vertex of $\mathcal{C}_{i}$ to every 
vertex of $\mathcal{C}_{t}$. We could connect every vertex of the base of $\mathcal{C}_{i}$ to every vertex $\mathcal{C}_{t}$ and make no other connections. We could connect every vertex of the summit of $\mathcal{C}_{i}$ to every vertex $\mathcal{C}_{t}$ and make no other connections. We could connect every vertex of the base of $\mathcal{C}_{i}$ to every vertex in the base of $\mathcal{C}_{t}$ and make no other connections, etc.. Of course, we can iterate this type of procedure. For example, replace the empty graph on $\mathcal{C}_{i}$ by one of the filtered digraphs that we just described.

\section{References}

[1] C.W. Borchardt, Ueber eine der Interpolation entsprechende Darstellung der Eliminations-Resultante, J. reine angew. Math. 57 (1860), pp. 111-121.

[2] C.J. Colborn, R.P.J. Day, and J.D. Nel, Unranking and Ranking Spanning Trees of a Graph, J. of Algorithms, 10, (1989), pp. 271-286.

[3] P. Doyle, personal communication.

[4] Ömer Eğecioğlu and Jeffrey B. Remmel, Bijections for Cayley Trees, Spanning Trees, and their q-Analogues, Journal of Combinatorial Theory, Series A, Vol. 42. No. 1 (1986), pp. 15-30.

[5] Ömer Eğecioğlu and Jeffrey B. Remmel, A bijection for spanning trees of complete multipartite graphs, Congress Numerautum 100 (1994), pp. 225-243.

[6] Ömer Eğecioğlu and Jeffrey B. Remmel, Ranking and Unranking Spanning Trees of Complete Multipartite Graphs, Preprint.

[7] Ö. Eğecioğlu and L.P. Shen, A Bijective Proof for the Number of Labeled q-trees, Ars Combinatoria 25B (1988), pp. 3-30.

[8] R. Onodera, Number of trees in the complete $N$-partite graph, RAAG Res. Notes $\mathbf{3}$, No. 192 (1973), pp. i +6 .

[9] H. Prufer, Never Bewies eines Satzes über Permutationen, Arch. Math. Phys. Sci. 27 (1918), pp. 742-744.

[10] A. Nijenhaus and H.S. Wilf, Combinatorial Algorithms, 2nd. ed., Academic Press, New York, (1978).

[11] E.M. Reingold, J. Neivergelt, and N. Deo, Combinatorial Algorithms: Theory and Practice, Prentice Hall, Englewood Cliffs, N.J., (1977)

[12] S.G. Williamson, Combinatorics for Computer Science, Computer Science Press, Rockville, MD., (1985). 